\title{
VIABILITY KERNELS AND CONTROL SETS
}

\author{
Dietmar SzOLNOKI ${ }^{1}$
}

\begin{abstract}
This paper analyzes the relation of viability kernels and control sets of control affine systems. A viability kernel describes the largest closed viability domain contained in some closed subset $Q$ of the state space. On the other hand, control sets are maximal regions of the state space where approximate controllability holds. It turns out that the viability kernel of $Q$ can be represented by the union of domains of attraction of chain control sets, defined relative to the given set $Q$. In particular, with this result control sets and their domains of attraction can be computed using techniques for the computation of attractors and viability kernels.
\end{abstract}

AMS Subject Classification. 34H05, 93B03.

Received December 10, 1998. Revised December 14, 1999.

\section{INTRODUCTION}

We consider the following class of control affine systems

$$
\begin{aligned}
\dot{x}(t) & =X_{0}(x(t))+\sum_{i=1}^{m} u_{i}(t) X_{i}(x(t)), \quad t \in \mathbb{R} \\
u \in \mathcal{U} & =\left\{u: \mathbb{R} \rightarrow \mathbb{R}^{m}, u(t) \in U \text { for almost all } t \in \mathbb{R}, \text { measurable }\right\}
\end{aligned}
$$

on a connected Riemannian $C^{\infty}$-manifold $M$ of dimension $d<\infty$, where $X_{0}, \ldots, X_{m}: M \rightarrow \mathbf{T} M$ are $C^{\infty}$-vector fields on $M$ and $U \subset \mathbb{R}^{m}$ is compact and convex.

We assume that for each $u \in \mathcal{U}$ and $x \in M$ the equation above has a unique solution $\varphi(t, x, u), t \in \mathbb{R}$, satisfying $\varphi(0, x, u)=x$. by

We supply $\mathcal{U}$ with the weak* topology of the dual space of $L_{1}\left(\mathbb{R}, \mathbb{R}^{m}\right), L_{\infty}\left(\mathbb{R}, \mathbb{R}^{m}\right)$, that is, a metric is given

$$
\mathrm{d}(u, v):=\sum_{n=1}^{\infty} \frac{1}{2^{n}} \frac{\left|\int_{\mathbb{R}}\left\langle u(t)-v(t), x_{n}(t)\right\rangle d t\right|}{1+\left|\int_{\mathbb{R}}\left\langle u(t)-v(t), x_{n}(t)\right\rangle d t\right|}, \quad u, v \in \mathcal{U}
$$

where $\left\{x_{n}, n \in \mathbb{N}\right\}$ is a countable, dense subset of $L_{1}\left(\mathbb{R}, \mathbb{R}^{m}\right)$ and $\langle\cdot, \cdot\rangle$ denotes the inner product in $\mathbb{R}^{m}$. With this metric, $\mathcal{U}$ is a compact, complete, separable metric space (see e.g. Colonius and Kliemann [4], Sect. 4.2).

Keywords and phrases: Control affine system, viability kernel, reachable set, control set, chain control set, control flow.

1 Universität Augsburg, Institut für Mathematik, Universitätsstraße 14, 86135 Augsburg, Germany;

e-mail: szolnoki@math.uni-augsburg.de 
Section 2 recalls the notion of viability kernels. A standard reference on viability theory is the book by Aubin [1], it deals with differential inclusions, a concept for differential equations with multiple right hand side. For a given closed subset $Q$ of the state space, the (positive) viability kernel is defined as the largest closed (positive) viability domain contained in $Q$. This concept is applied to the control system (1.1), and also introduced for negative time direction, and for the complete time axes, which yields the definition of the negative viability kernel of $Q$, and the $\mathbb{R}$-viability kernel of $Q$. The $\mathbb{R}$-viability kernel can be represented as the set of all initial points in $Q$ having the property that there exists some control function such that the corresponding trajectory remains in $Q$ for all times. In particular, the $\mathbb{R}$-viability kernel is given by the intersection of the positive and negative viability kernel.

Section 3 analyzes the relation of viability kernels and control sets. The concept of control sets was introduced in Kliemann [7]. Control sets are maximal regions of the state space where approximate controllability holds. A good introduction to control sets is Häckl [6], and a comprehensive theory on control sets can be found in [4] Chapter 3. First we recall the notion of local accessibility and state a condition under which local accessibility holds. This condition is based on the Lie algebra generated by the vector fields of the control system (see $e . g$. Nijmeijer and van der Schaft [8]). Furthermore (from [4], Sect. 3.2) we state some properties of control sets under a local accessibility condition, which yield a first connection of viability kernels and control sets: a control set in a closed subset $Q$ is contained in the $\mathbb{R}$-viability kernel of $Q$.

To obtain a more precise result, we introduce an appropriate notion of chain control sets and their domains of attraction relative to $Q$. If $Q$ is the whole state space, the relative concept coincides with the standard concept of chain control sets and their domains of attraction. It can be shown that chain control sets correspond to maximal chain transitive subsets, that is (connected) chain recurrent components of the corresponding control flow, see Colonius and Kliemann ([3], Sect. 4). As a consequence, all compact $\omega$-limit sets are contained in chain control sets. Using this result, it finally turns out that the viability kernel of a compact subset $Q$ of the state space can be represented by the union of the relative domains of attraction of all relative chain control sets.

With these results we are now in the position to use algorithms for the computation of viability kernels also for the computation of control sets and their domains of attraction. Such an algorithm has been developed by Saint-Pierre [9] and [10]. In Section 4 we consider as an example a system modelling a continuous stirred chemical tank reactor. The corresponding pictures have been created using the idea from Saint-Pierre and an implementation of a subdivision algorithm for the computation of attractors, unstable manifolds and invariant measures of discrete time dynamical systems (GAIO), see Dellnitz and Hohmann [5]. The subdivision algorithm for the computation of viability kernels of discrete time control systems is discussed in Szolnoki [11].

It should be noted that there exists an excellent algorithm (and its implementation "CS") for the computation of control sets, see Häckl [6] (or [4], Append. C). However, the efficiency of this algorithm decreases dramatically in dimensions higher than two. We expect that subdivision techniques are better suited for higher dimensions. This is part of present research.

\section{Viability KERNELS}

This section briefly recalls the notion of viability domains and viability kernels. For more detailed information we refer to Aubin [1]. For our purpose it is convenient to consider both positive and negative time direction.

Definition 2.1. A set $S \subset M$ is called a positive viability domain of the system (1.1), if for all $x \in S$ there is a control function $u \in \mathcal{U}$, such that the solution $\varphi(t, x, u) \in S$ for all $t \geq 0$. A set $S \subset M$ is called a negative viability domain of the system (1.1), if for all $x \in S$ there is a control function $u \in \mathcal{U}$, such that the solution $\varphi(t, x, u) \in S$ for all $t \leq 0$. A set which is a positive and negative viability domain is called a $\mathbb{R}$-viability domain.

We now consider viability domains contained in some closed subset $Q$. More precisely, we are interested in the largest closed viability domain contained in $Q$.

Definition 2.2. Let $Q$ be a closed subset of $M$. The largest closed positive viability domain in $Q$ is called the positive viability kernel of $Q$ for the system (1.1) and is denoted by $\operatorname{Viab}_{Q}^{+}$. The largest closed negative viability 
domain in $Q$ is called the negative viability kernel of $Q$ for the system (1.1) and is denoted by $\mathrm{Viab}_{Q}^{-}$, and the largest closed $\mathbb{R}$-viability domain in $Q$ is called the $\mathbb{R}$-viability kernel of $Q$ for the system (1.1) and is denoted by $\operatorname{Viab}_{Q}^{\mathbb{R}}$.

The following theorem, which has been proved in [1], Section 4.1, in a more general context, states that the viability kernel is given by all initial points viable in $Q$.

Theorem 2.3. Let $Q \subset M$ be closed. Then the positive viability kernel of $Q$ is equal to the subset of all initial states $x \in Q$ having the property that there exists at least one control function $u \in \mathcal{U}$ such that $\varphi(t, x, u) \in Q$ for all $t \geq 0$. The negative viability kernel of $Q$ is equal to the subset of all initial states $x \in Q$ having the property that there exists at least one control function $u \in \mathcal{U}$ such that $\varphi(t, x, u) \in Q$ for all $t \leq 0$, and the $\mathbb{R}$-viability kernel of $Q$ is equal to the subset of all initial states $x \in Q$ having the property that there exists at least one control function $u \in \mathcal{U}$ such that $\varphi(t, x, u) \in Q$ for all $t \in \mathbb{R}$.

Proof. We define

$$
\begin{aligned}
& \operatorname{Viab}_{Q}^{+}:=\{x \in Q, \text { there exists } u \in \mathcal{U} \text { such that } \varphi(t, x, u) \in Q \text { for all } t \geq 0\} \\
& \operatorname{Viab}_{Q}^{-}:=\{x \in Q, \text { there exists } u \in \mathcal{U} \text { such that } \varphi(t, x, u) \in Q \text { for all } t \leq 0\}, \\
& \operatorname{Viab}_{Q}^{\mathbb{R}}:=\{x \in Q, \text { there exists } u \in \mathcal{U} \text { such that } \varphi(t, x, u) \in Q \text { for all } t \in \mathbb{R}\} .
\end{aligned}
$$

For $\operatorname{Viab}_{Q}^{+}$we check the properties required in Definition 2.2. $\operatorname{Viab}_{Q}^{-}$and $\mathrm{Viab}_{Q}^{\mathbb{R}}$ have to be treated analogously.

Closedness: Let $x \in \operatorname{clViab}_{Q}^{+}$. Choose a sequence $\left(x_{j}\right) \subset \mathrm{Viab}_{Q}^{+}$converging to $x$. For every $x_{j}$ there is a control function $u_{j} \in \mathcal{U}$, such that $\varphi\left(t, x_{j}, u_{j}\right) \in Q$ for all $t \geq 0$. Since $\mathcal{U}$ is compact with the metric (1.2), there exists a convergent subsequence of $\left(u_{j}\right) \subset \mathcal{U}$. We denote this subsequence again by $\left(u_{j}\right)$, converging to $u \in \mathcal{U}$. In [2], proof of Lemma 3.4, it has been proved that, for all $t \geq 0$ the sequence $\varphi\left(t, x_{j}, u_{j}\right)$ converges to $\varphi(t, x, u)$. By compactness of $Q, \varphi(t, x, u) \in Q$ for all $t \geq 0$. Hence, by definition, $x \in \mathrm{Viab}_{Q}^{+}$, and we obtain $\mathrm{cl} \mathrm{Viab}_{Q}^{+} \subseteq \operatorname{Viab}_{Q}^{+}$.

Viability: Let $x \in \operatorname{Viab}_{Q}^{+}$. By definition, there is a control function $u \in \mathcal{U}$ such that $\varphi(t, x, u) \in Q$ for all $t \geq 0$. For arbitrary $\tau \geq 0$ choose the shifted control function $u^{\prime}:=u(\tau+\cdot)$, that is, $\varphi\left(t, \varphi(\tau, x, u), u^{\prime}\right) \in Q$ for all $t \geq 0$, hence $\varphi(\tau, x, u) \in \operatorname{Viab}_{Q}^{+}$for all $\tau \geq 0$, that is, $\operatorname{Viab}_{Q}^{+}$is a positive viability domain.

Maximality: Let $S \subset Q$ be a closed positive viability domain. Thus, for all $x \in S$ there exists $u \in \mathcal{U}$ with $\varphi(t, x, u) \in S \subset Q$ for all $t \geq 0$, that is, $x \in \operatorname{Viab}_{Q}^{+}$.

The $\mathbb{R}$-viability kernel is given by the intersection of the positive and negative viability kernel,

$$
\operatorname{Viab}_{Q}^{\mathbb{R}}=\operatorname{Viab}_{Q}^{+} \cap \operatorname{Viab}_{Q}^{-} .
$$

To see this, choose $x \in \operatorname{Viab}_{Q}^{+} \cap \operatorname{Viab}_{Q}^{-}$. By Theorem 2.3, there are control functions $u_{1}, u_{2} \in \mathcal{U}$ such that $\varphi\left(t, x, u_{1}\right) \in Q$ for all $t \geq 0$ and $\varphi\left(t, x, u_{2}\right) \in Q$ for all $t \leq 0$. Defining $u \in \mathcal{U}$ as a concatenation of $\left.u_{1}\right|_{[0, \infty]}$ and $\left.u_{2}\right|_{(-\infty, 0)}$, it immediately follows $x \in \operatorname{Viab}_{Q}^{\mathbb{R}}$. The other inclusion is obvious.

\section{ViABILITY KERNELS AND CONTROL SETS}

This section deals with the relation of the viability kernel of a closed subset $Q$ and control sets of the underlying system. Control sets are maximal regions of the state space where it is possible to steer from every point to every other point, at least approximately, see Colonius and Kliemann ([4], Sect. 3.1).

As a first observation we obtain that, under a local accessibility condition, control sets $D$ in $Q$ having nonvoid interior are also contained in the $\mathbb{R}$-viability kernel of $Q$. For a better insight we use the concept of chain controllability: chain control sets are maximal subsets of the state space where any two points can be connected by chains (see [4], Sect. 3.1). We introduce a notion of chain control sets and their domains of 
attraction relative to the closed subset $Q$, and it turns out that this is the appropriate definition to describe the relation of viability kernels and control sets. For that purpose we consider a dynamical system attached to the control affine system (1.1), called the control flow. The control system can be viewed as a family of differential equations, indexed by the control functions $u \in \mathcal{U}$. The control flow encompasses all these differential equations. In [4], Section 4.3, it has been proved that chain control sets coincide with the projection of maximal chain transitive subsets of the corresponding control flow. As a consequence, all compact $\omega$-limit sets are contained in chain control sets. Using these results, we prove that the viability kernel of $Q$ can be represented by the union of the relative domains of attraction of all chain control sets relative to $Q$.

Let $\mathcal{O}_{\leq T}^{+}(x)$ and $\mathcal{O}_{\leq T}^{-}(x)$ denote the positive reachable set of $x$ up to time $T>0$ and the negative reachable set of $x$ up to time $T>0$, respectively. Furthermore, let $\mathcal{O}^{+}(x)$ and $\mathcal{O}^{-}(x)$ denote the positive reachable set of $x$ and the negative reachable set of $x$, respectively. The control system (1.1) is called locally accessible if for all $x \in M$

$$
\operatorname{int} \mathcal{O}_{\leq T}^{+}(x) \neq \emptyset \quad \text { and } \quad \operatorname{int} \mathcal{O}_{\leq T}^{-}(x) \neq \emptyset \quad \text { for all } T>0
$$

Recall e.g. from Nijmeijer and van der Schaft [8] that local accessibility is guaranteed by the following accessibility rank condition

$$
\operatorname{dim} \mathcal{L} \mathcal{A}\left\{X_{0}+\sum_{i=1}^{m} u_{i} X_{i},\left(u_{i}\right) \in U\right\}(x)=d \quad \text { for all } x \in M,
$$

where $\mathcal{L} \mathcal{A}$ denotes the Lie algebra generated by the corresponding vector fields.

The following proposition states some properties of control sets under a local accessibility condition.

Proposition 3.1. If the system (1.1) is locally accessible and $D \subset M$ a control set with nonvoid interior, then

(i) $\operatorname{clint} D=\operatorname{cl} D$,

(ii) int $D \subset \mathcal{O}^{+}(x)$ for every $x \in D$, that is, in int $D$ we have controllability,

(iii) $D=\operatorname{clO}^{+}(x) \cap \mathcal{O}^{-}(x)$ for every $x \in \operatorname{int} D$.

For the proof we refer to [4], Section 3.2. As a consequence, we obtain that control sets $D$ in $Q$ having nonvoid interior are also contained in the $\mathbb{R}$-viability kernel of $Q$ :

Corollary 3.2. Assume that the system (1.1) is locally accessible, and let $D \subset Q$ be a control set with nonvoid interior. Then,

$$
\operatorname{cl} D \subseteq \operatorname{Viab}_{Q}^{\mathbb{R}}
$$

Proof. Choose $x$ and $y$ in int $D$. From Proposition 3.1(ii) we know that in int $D$ we have controllability, that is, there exist finite trajectories from $x$ to $y$ and from $y$ to $x$. Thus we have constructed a periodic trajectory $\varphi$ containing $x$ and $y$. For every point $z \in \varphi$ we have $z \in \mathcal{O}^{+}(x)$ and $z \in \mathcal{O}^{-}(x)$, and by Proposition 3.1(iii) follows $z \in D$, that is, $\varphi \subset D$. It follows immediately int $D \subset \operatorname{Viab}_{Q}^{\mathbb{R}}$. Since $\operatorname{Viab}_{Q}^{\mathbb{R}}$ is closed, we obtain from Proposition 3.1(i) cl int $D=\operatorname{cl} D \subseteq \operatorname{Viab}_{Q}^{\mathbb{R}}$.

We now introduce the notion of $(\varepsilon, T)$-chains from the theory of dynamical systems. Recall that a flow or continuous dynamical system on a metric space $X$ is given by a continuous map $\Psi: \mathbb{R} \times X \rightarrow X$ which satisfies $\Psi(0, x)=x$ and $\Psi(t+s, x)=\Psi(t, \Psi(s, x))$ for all $x \in X$ and all $t, s \in \mathbb{R}$.

Definition 3.3. Let $\Psi$ be a flow on a compact metric space $X$. For $x, y \in X$ and $\varepsilon, T>0$ a $(\varepsilon, T)$-chain from $x$ to $y$ is given by $n \in \mathbb{N}$, together with points $x_{0}, \ldots, x_{n} \in X$ and times $T_{0}, \ldots, T_{n-1} \geq T$ with $x_{0}=x, x_{n}=y$, and

$$
\mathrm{d}\left(\Psi\left(T_{i}, x_{i}\right), x_{i+1}\right) \leq \varepsilon \quad \text { for all } i=0, \ldots, n-1 .
$$

The $\omega$-limit set and the $\alpha$-limit set of a point $x \in X$ are defined as usual,

$$
\begin{aligned}
\omega(x) & :=\left\{y \in X, \text { there are } t_{k} \rightarrow \infty \text { with } \Psi\left(t_{k}, x\right) \rightarrow y\right\}, \\
\alpha(x) & :=\left\{y \in X, \text { there are } t_{k} \rightarrow-\infty \text { with } \Psi\left(t_{k}, x\right) \rightarrow y\right\}
\end{aligned}
$$


Definition 3.4. A flow on a metric space $X$ is called topologically transitive, if there exists some $x \in X$ such that $\omega(x)=X$.

A subset $Y \subset X$ is chain transitive if for all $x, y \in Y$ and all $\varepsilon, T>0$ there exists a $(\varepsilon, T)$-chain from $x$ to $y$.

Proposition 3.5. If the flow is topologically transitive, then it is chain transitive. In other words, a flow restricted to an $\omega$-limit set $\omega(x)$ with $x \in X$ is chain transitive.

The proof is given e.g. in [4], Appendix B.2. We now transfer the notion of $(\varepsilon, T)$-chains of dynamical systems to control systems, in particular, to the control system (1.1).

Definition 3.6. Fix $x, y \in M$ and let $\varepsilon, T>0$. A controlled $(\varepsilon, T)$-chain from $x$ to $y$ is given by $n \in \mathbb{N}$, $x_{0}, \ldots, x_{n} \in M, u_{0}, \ldots, u_{n-1} \in \mathcal{U}$ and $t_{0}, \ldots, t_{n-1} \geq T$ with $x_{0}=x, x_{n}=y$, and

$$
\mathrm{d}\left(\varphi\left(t_{j}, x_{j}, u_{j}\right), x_{j+1}\right) \leq \varepsilon \quad \text { for all } j=0, \ldots, n-1 .
$$

If for every $\varepsilon, T>0$ there is an $(\varepsilon, T)$-chain from $x$ to $y$, then the point $x$ is chain controllable to $y$.

For a point $x \in M$ the $\omega$-limit set and the $\alpha$-limit set, respectively, with respect to a control function $u \in \mathcal{U}$ are defined by

$$
\begin{aligned}
\omega(u, x) & :=\left\{y \in M, \text { there are } t_{k} \rightarrow \infty \text { with } \varphi\left(t_{k}, x, u\right) \rightarrow y\right\} \\
\alpha(u, x) & :=\left\{y \in M, \text { there are } t_{k} \rightarrow-\infty \text { with } \varphi\left(t_{k}, x, u\right) \rightarrow y\right\}
\end{aligned}
$$

Since we are interested in the relation to viability kernels, which are defined with respect to closed subsets of the state space, we introduce a notion of chain control sets and their domains of attraction relative to some given closed subset $Q \subset M$.

Definition 3.7. A set $E_{Q} \subset Q$ is called a chain control set relative to $Q$, if

(i) $E_{Q}$ is a $\mathbb{R}$-viability domain,

(ii) for all $x, y \in E_{Q}$ and $\varepsilon, T>0$ there is a controlled $(\varepsilon, T)$-chain from $x$ to $y$, which is completely contained in $Q$, that means $\varphi\left(t, x_{j}, u_{j}\right) \in Q$ for all $t \in\left[0, t_{j}\right], j=0, \ldots, n-1$,

(iii) $E_{Q}$ is maximal (with respect to set inclusion) with these properties.

For these relative chain control sets we also define their relative domains of attraction.

Definition 3.8. For a chain control set $E_{Q}$ relative to $Q$, the positive domain of attraction relative to $Q$ is defined by

$$
\mathbf{A}_{Q}^{+}\left(E_{Q}\right):=\left\{x \in Q, \text { there is } u \in \mathcal{U} \text { with } \varphi(t, x, u) \in Q \text { for all } t \geq 0 \text { and } \emptyset \neq \omega(u, x) \subset E_{Q}\right\}
$$

and the negative domain of attraction relative to $Q$ is defined by

$$
\mathbf{A}_{Q}^{-}\left(E_{Q}\right):=\left\{x \in Q \text {, there is } u \in \mathcal{U} \text { with } \varphi(t, x, u) \in Q \text { for all } t \leq 0 \text { and } \emptyset \neq \alpha(u, x) \subset E_{Q}\right\}
$$

Note that in the case when $Q$ is the whole state space, the definitions of chain control sets and their domains of attraction relative to $Q$ coincide with the standard definitions of chain control sets and their domains of attraction given in [4], Section 3.1. Furthermore note that a chain control set $E$ is, by definition, a $\mathbb{R}$-viability domain and, in particular, closed (see [4], Sect. 4.3). Hence, from the maximality property of viability kernels, it immediately follows $\operatorname{Viab}_{E}^{\mathbb{R}}=E$. The same holds true for relative chain control sets $E_{Q}$, i.e. Viab $E_{E_{Q}}^{\mathbb{R}}=E_{Q}$.

We now attach a dynamical system to the given control system (1.1), called the control flow. It is given by the following map

$$
\Phi: \mathbb{R} \times \mathcal{U} \times M \rightarrow \mathcal{U} \times M, \quad \Phi(t, u, x):=(\Theta(t, u), \varphi(t, x, u))
$$


where $\Theta$ denotes the shift on $\mathcal{U}$

$$
\Theta: \mathbb{R} \times \mathcal{U} \rightarrow \mathcal{U}, \quad \Theta_{t}(u):=\Theta(t, u):=u(t+\cdot),
$$

where $u(t+\cdot)(s):=u(t+s), s \in \mathbb{R}$. Indeed, the map $\Phi$ defines a continuous dynamical system on $\mathcal{U} \times M$ (see [4], Sect. 4.3). The following proposition clarifies the relation of chain control sets of the control system (1.1) and chain transitive sets of the corresponding control flow $(\mathcal{U} \times M, \Phi)$.

Proposition 3.9. Let $E \subset M$ be a chain control set of the system (1.1). Then the lift of $E$

$$
\mathcal{E}:=\{(u, x) \in \mathcal{U} \times M, \varphi(t, x, u) \in E \text { for all } t \in \mathbb{R}\}
$$

is a maximal (with respect to set inclusion) invariant and chain transitive set for the control flow $(\mathcal{U} \times M, \Phi)$.

Conversely, let $\mathcal{E} \subset \mathcal{U} \times M$ be a maximal invariant and chain transitive set for the control flow. Then the projection of $\mathcal{E}$ onto $M$

$$
\pi_{M}(\mathcal{E}):=\{x \in M, \text { there exists } u \in \mathcal{U} \text { such that }(u, x) \in \mathcal{E}\}
$$

is a chain control set.

For the proof we refer to Colonius and Kliemann [3], Section 4. Since $\omega$-limit sets of the control flow are chain transitive, see Proposition 3.5, we obtain as an immediate corollary to Proposition 3.9 that $\omega$-limit sets of the corresponding control system are contained in chain control sets. Note that the same statement can be formulated for $\alpha$-limit sets by defining chains with negative times.

Corollary 3.10. For every $(u, x) \in \mathcal{U} \times M$ with bounded positive trajectory $\varphi(t, x, u), t \geq 0$, there exists a chain control set $E$ such that the $\omega$-limit set of $x$ with respect to the control function $u$ is contained in $E$, i.e., $\omega(u, x) \subset E$.

Now we are in the position to give an equivalent representation for viability kernels.

Theorem 3.11. Let $Q \subset M$ be compact. Then,

$$
\operatorname{Viab}_{Q}^{+}=\bigcup_{E_{Q}} \mathbf{A}_{Q}^{+}\left(E_{Q}\right) \quad \text { and } \quad \operatorname{Viab}_{Q}^{-}=\bigcup_{E_{Q}} \mathbf{A}_{Q}^{-}\left(E_{Q}\right),
$$

where the union is taken over all chain control sets $E_{Q}$ relative to $Q$.

Proof. The claim is proved only for positive viability kernels, negative viability kernels have to be treated analogously.

"ᄃ": Let $z \in \operatorname{Viab}_{Q}^{+}$, that is, there is a control function $u \in \mathcal{U}$ such that $\varphi(t, z, u) \in Q$ for all $t \geq 0$. By compactness of $Q$, the $\omega$-limit set $\omega(u, z)$ is contained in $Q$ and nonvoid. By Corollary 3.10, $\omega(u, z)$ is contained in some chain control set. We claim that the controlled $(\varepsilon, T)$-chains connecting any two points of $\omega(u, z)$ can be chosen such that they are completely contained in $\omega(u, z) \subset Q$. Hence, $\omega(u, z)$ is contained in some relative chain control set $E_{Q}$, and it immediately follows $z \in \mathbf{A}_{Q}^{+}\left(E_{Q}\right)$.

The claim is proved as follows. We consider the lift of $\omega(u, z)$, that is, the $\omega$-limit $\operatorname{set} \omega((u, z))$ of $(u, z) \subset \mathcal{U} \times M$ for the corresponding control flow $(\mathcal{U} \times M, \Phi)$. By Proposition 3.5, the control flow restricted to $\omega((u, z))$ is chain transitive, that is, the $(\varepsilon, T)$-chains connecting some two points in $\omega((u, z))$ can be chosen such that they are completely contained in $\omega((u, z))$. Projecting down these chains onto $M$, one obtains controlled $(\varepsilon, T)$-chains, connecting any two points of $\omega(u, z)$, which are completely contained in $\omega(u, z) \subset Q$. 
"卫": By construction, the relative positive domain of attraction of any relative chain control set is positively viable in $Q$, hence contained in $\operatorname{Viab}_{Q}^{+}$.

From Section 2 we know that the $\mathbb{R}$-viability kernel of $Q$ is given by the intersection of the positive and the negative viability kernel of $Q$, see formula (2.3). Hence we obtain

$$
\operatorname{Viab}_{Q}^{\mathbb{R}}=\bigcup_{E_{Q}} \mathbf{A}_{Q}^{+}\left(E_{Q}\right) \cap \bigcup_{E_{Q}} \mathbf{A}_{Q}^{-}\left(E_{Q}\right) .
$$

Remark 3.12. In [4], Section 4.7, the analysis of control systems with perturbed control range yields that, under local accessibility and inner pair conditions, control sets and chain control sets generically coincide. If $Q$ is the whole state space, then we obtain

$$
\operatorname{Viab}_{Q}^{\mathbb{R}}=\bigcup_{D} \operatorname{cl} \mathbf{A}^{+}(D) \cap \bigcup_{D} \operatorname{cl} \mathbf{A}^{-}(D)
$$

"almost always" in the sense of above mentioned analysis.

\section{ExAmple}

The analogous results on the relation of viability kernels and control sets can also be obtained for discrete time control systems

$$
x_{n+1}=f\left(x_{n}, u_{n}\right), \quad n \in \mathbb{Z} .
$$

Furthermore, an algorithm for the computation of viability kernels of recursive inclusions

$$
x_{n+1} \in F\left(x_{n}\right), \quad n \in \mathbb{N},
$$

where $F$ denotes a set valued map (see Saint-Pierre [9]), can be adapted to control systems (4.4), see Szolnoki [11]. This algorithm has been implemented by modifying an existing implementation of a subdivision algorithm ("GAIO" by Dellnitz and Junge) for the computation of unstable manifolds, invariant measures and, in particular, relative global attractors of discrete time dynamical systems, see Dellnitz and Hohmann [5].

The relation of viability kernels and attractors is as follows. Recall that for a flow $\Psi$ on a compact metric space $X$, a set $A \subset X$ is called an attractor, if it admits a neighborhood $V \supset A$ such that

$$
A=\bigcap_{t \geq 0} \Psi(t, V)
$$

If $V$ is the whole state space, then $A$ is called global attractor for $(X, \Psi)$. Accordingly, for a compact subset $K \subset X$, the set

$$
A_{K}:=\bigcap_{t \geq 0} \Psi(t, K)
$$

is called global attractor relative to $K$. Note that this definition of a relative attractor has been introduced in Dellnitz and Hohmann [5] for discrete time dynamical systems.

Proposition 4.1. Let $Q \subset M$ be compact. Then the lift of the negative viability kernel of $Q$, defined as

$$
\mathcal{V}:=\{(u, x) \in \mathcal{U} \times M, \varphi(t, x, u) \in Q \text { for all } t \leq 0\}
$$

is the global attractor relative to $\mathcal{U} \times Q$ for the control flow $(\mathcal{U} \times M, \Phi)$. 
Conversely, let $\mathcal{V}$ be the global attractor relative to $\mathcal{U} \times Q$ for the control flow. Then the projection of $\mathcal{V}$ onto $M$

$$
\pi_{M}(\mathcal{V}):=\{x \in M, \text { there exists } u \in \mathcal{U} \text { such that }(u, x) \in \mathcal{V}\}
$$

is the negative viability kernel of $Q$.

Proof. This is immediate from the definitions.

The idea of the algorithm for discrete time systems (4.4) is to approximate the $\mathbb{R}$-viability kernel of $Q$ by iterative subdivision and selection steps. Starting with $\mathcal{B}_{0}:=\{Q\}$ one obtains $\mathcal{B}_{k}$ from $\mathcal{B}_{k-1}$ in two steps:

(i) subdivision step: Construct a family $\hat{\mathcal{B}}_{k}$ such that

$$
\bigcup_{B \in \hat{\mathcal{B}}_{k}} B=\bigcup_{B \in \mathcal{B}_{k-1}} B
$$

and

$$
\operatorname{diam}\left(\hat{\mathcal{B}}_{k}\right)=\theta_{k} \operatorname{diam}\left(\mathcal{B}_{k-1}\right)
$$

with $0<\theta_{\min } \leq \theta_{k} \leq \theta_{\max }<1$.

(ii) Selection step: Define $\mathcal{B}_{k}$ by

$$
\mathcal{B}_{k}:=\left\{B \in \hat{\mathcal{B}}_{k} \mid \exists u_{1}, u_{2} \in U \exists \hat{B}_{1}, \hat{B}_{2} \in \hat{\mathcal{B}}_{k}: f_{u_{1}}^{-1}(B) \cap \hat{B}_{1} \neq \emptyset \wedge f_{u_{2}}(B) \cap \hat{B}_{2} \neq \emptyset\right\} .
$$

Note that using this algorithm for the computation of $\mathbb{R}$-viability kernels of continuous time control systems (1.1) requires to overcome problems concerning the refinement of spatial, time and control range discretization. These problems are part of present research. For differential inclusions, related questions are discussed in Saint-Pierre [10]. In particular, it turns out that the refinement of space and time has to be treated consistently to obtain a convergent computation scheme.

As an example, consider the following model of a continuous stirred chemical tank reactor

$$
\begin{aligned}
& \dot{x}=-x+B \alpha(1-y) e^{x}-u(t)\left(x-x_{c}\right) \\
& \dot{y}=-y+\alpha(1-y) e^{x}
\end{aligned}
$$

(see Uppal et al. [12]). In this simplified model, $x$ is the dimensionless temperature and $y$ the product concentration in the reactor. $x_{c}$ is the coolant temperature and $\alpha$ and $B$ are technical constants. The flow rate of the coolant liquid is modeled as the control input $u(t)$.

In Häckl ([6], Sect. 5.6), the system has been examined for $\alpha=0.05, B=10, x_{c}=1$. There are two stable and one hyperbolic equilibrium, see Figure 4.1. If the control function $u(t)$ takes values in the control range $U=[0.9,1.0]$, two invariant control sets (C1 and $\mathrm{C} 2)$, and one variant control set (C3), arise around the two stable equilibria, and the one hyperbolic equilibrium, respectively. The pictures in Figures 4.2-4.5 have been created with above mentioned algorithm. The green and blue lines represent the phase portraits for the two extremal controls $u \equiv 0.9$ (green) and $u \equiv 1.0$ (blue). Figure 4.2 and Figure 4.3 show approximations of the positive viability kernel of $Q=[2.5,3] \times[0.4,0.5]$, and the negative viability kernel of $Q$, respectively. The positive viability kernel coincides with the variant control set C3, including the part of the positive domain of attraction contained in $Q$. Analogously, the negative viability kernel coincides with the variant control set C3, including the part of the negative domain of attraction contained in $Q$. Figure 4.4 shows the $\mathbb{R}$-viability kernel of $Q$, that is, the intersection of the positive and the negative viability kernel of $Q$. Indeed, the $\mathbb{R}$-viability kernel coincides with the variant control set C3.

Figure 4.5 shows an approximation of the negative viability kernel of $Q=[2.5,3] \times[0.4,0.5]$. It coincides with the corresponding invariant control set $\mathrm{C} 1$. 


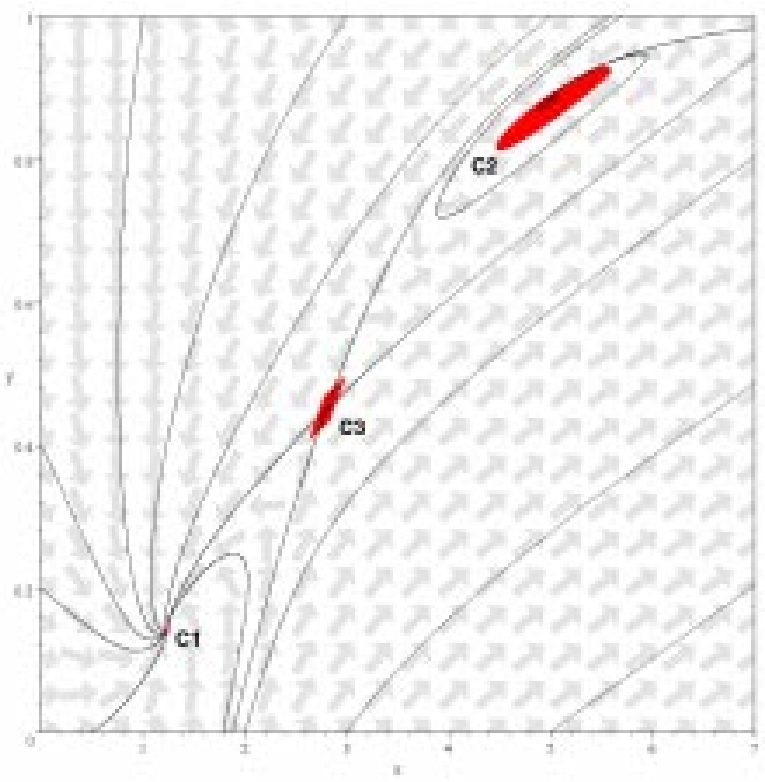

FIgURE 4.1. Phase portrait of the continuous stirred tank reactor, with invariant control sets $\mathrm{C} 1$ and $\mathrm{C} 2$ and variant control set $\mathrm{C} 3$ for control range $U=[0.9,1.0]$.

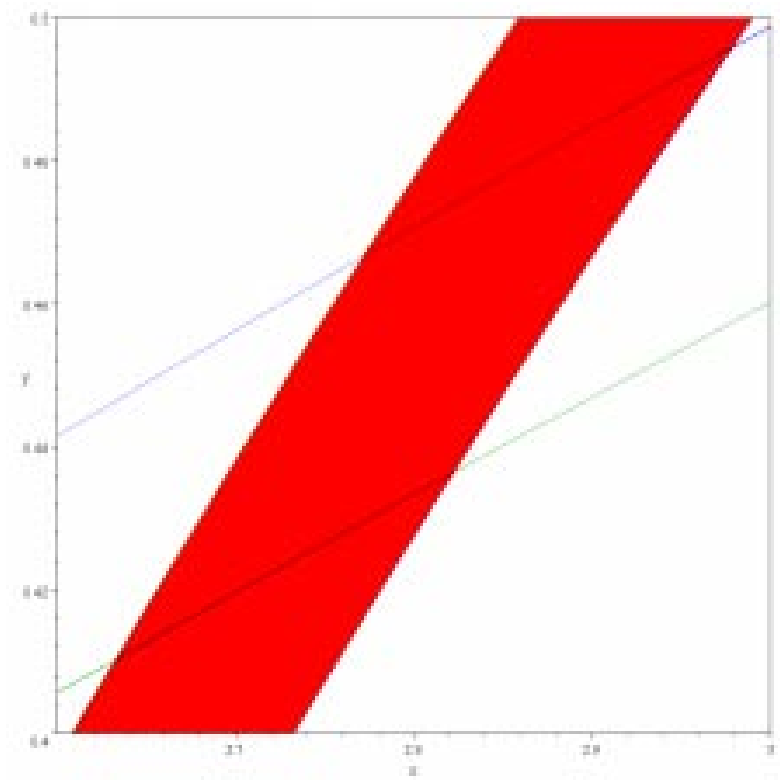

Figure 4.2. Approximation of the positive viability kernel of $Q=[2.5,3] \times[0.4,0.5]$. 


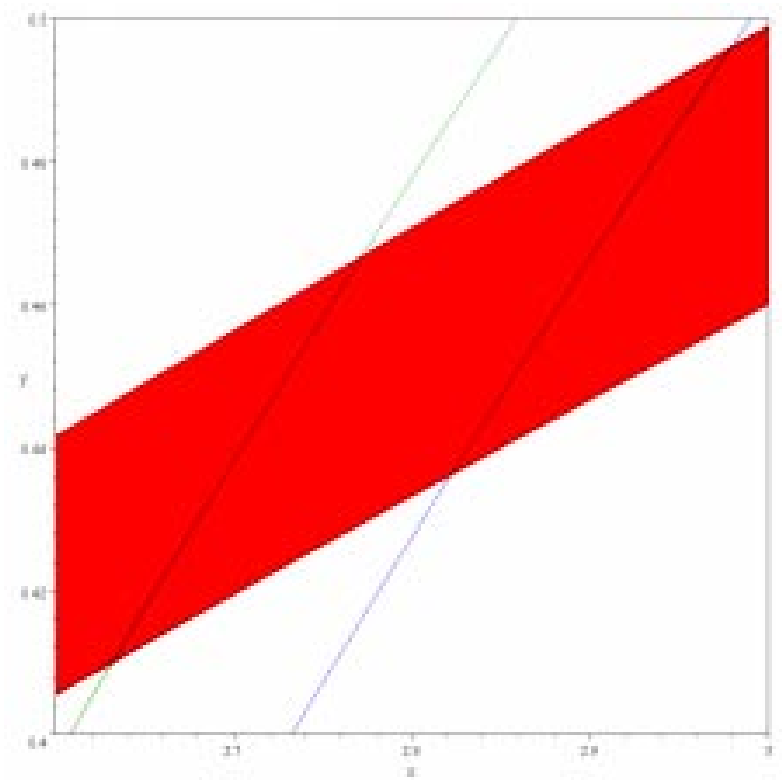

Figure 4.3. Approximation of the negative viability kernel of $Q=[2.5,3] \times[0.4,0.5]$.

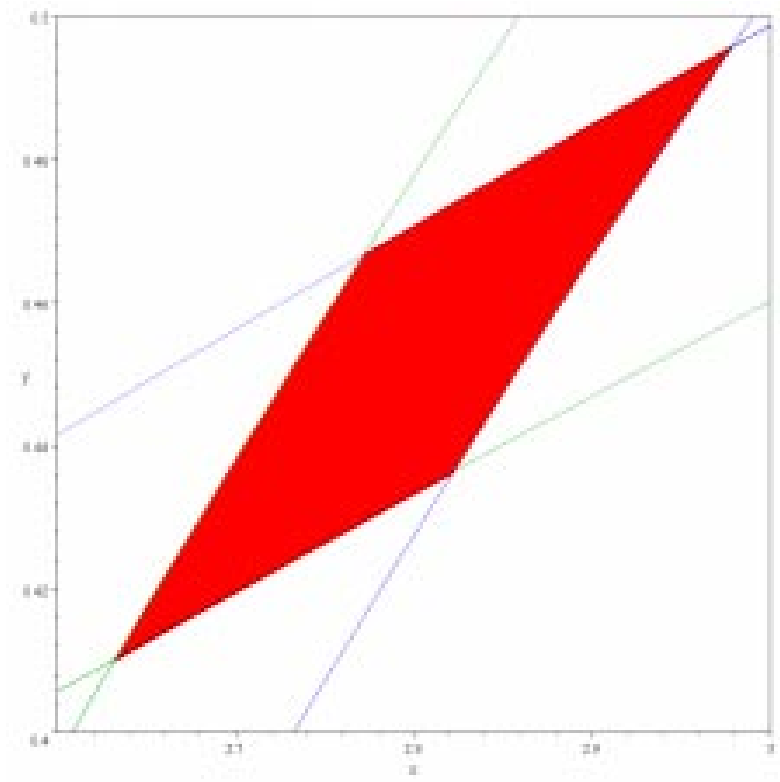

FiguRE 4.4. Approximation of the viability kernel of $Q=[2.5,3] \times[0.4,0.5]$. 


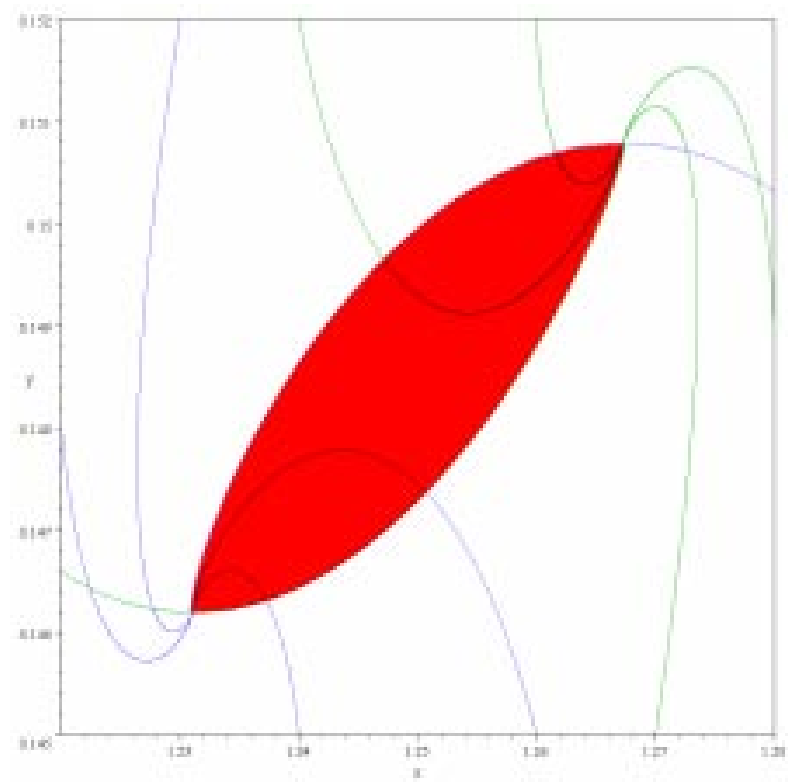

FIGURE 4.5. Approximation of the negative viability kernel of $Q=[1.2,1.3] \times[0.140,0.154]$.

I would like to thank Fritz Colonius for his constant help and numerous helpful discussions. Furthermore, I am grateful to Michael Dellnitz for making available the source code of 'GAIO' and Oliver Junge for his constant help concerning the software installation.

\section{REFERENCES}

[1] J.-P. Aubin, Viability Theory. Birkhäuser (1991).

[2] F. Colonius and W. Kliemann, Infinite time optimal control and periodicity. Appl. Math. Optim. 20 (1989) 113-130.

[3] - Some aspects of control systems as dynamical systems. J. Dynam. Differential Equations 5 (1993) 469-494.

[4] - The Dynamics of Control. Birkhäuser (2000) to appear.

[5] M. Dellnitz and A. Hohmann, A subdivision algorithm for the computation of unstable manifolds and global attractors. Numer. Math. 75 (1997) 293-317.

[6] G. HÄCKL, Reachable Sets, Control Sets and Their Computation. Dissertation, Universität Augsburg, "Augsburger Mathematische Schriften Band 7" (1996).

[7] W. KliEmann, Qualitative Theorie Nichtlinearer Stochastischer Systeme. Dissertation, Universität Bremen (1980).

[8] H. Nijmeijer and A.J. van der Schaft, Nonlinear Dynamical Control Systems. Springer-Verlag (1990).

[9] P. Saint-Pierre, Approximation of the viability kernel. Appl. Math. Optim. 29 (1994) 187-209.

[10] - Set-valued numerical analysis for optimal control and differential games (1998) to appear.

[11] D. Szolnoki, Berechnung von Viabilitätskernen. Diplomarbeit, Institut für Mathematik, Universität Augsburg, Augsburg (1997).

[12] A. Uppal, W.H. Ray and A.B. Poore, On the dynamic behavior of continuous stirred tank reactors. Chem. Engrg. Sci. 19 (1974) 967-985. 